\title{
Research on Dynamic Monitoring Algorithm of Visual Safety Distance in Highway
}

\author{
Jiajia Zhang, Yu Liu, Jianming Li, Yanhua Guan \\ Meteorological Technology and Equipment Center in Hebei Province, Shijiazhuang, China \\ Email: sunyi_12_12@sina.com
}

How to cite this paper: Zhang, J.J., Liu, Y., Li, J.M. and Guan, Y.H. (2016) Research on Dynamic Monitoring Algorithm of Visual Safety Distance in Highway. Journal of Geoscience and Environment Protection, 4, 612.

http://dx.doi.org/10.4236/gep.2016.411002

Received: October 17, 2016

Accepted: October 28, 2016

Published: October 31, 2016

Copyright $\odot 2016$ by authors and Scientific Research Publishing Inc. This work is licensed under the Creative Commons Attribution International License (CC BY 4.0).

http://creativecommons.org/licenses/by/4.0/

\begin{abstract}
To develop the dynamic monitoring algorithm of visual safety distance in highway, by using the highway video traffic monitoring system, the research platform of four kinds of terrain environment in plateau, mountainous area, plain and coastal area is established. Results show that through the contrast between the sample data and visibility train of thought, based on the theory of mathematical morphology, expressway visibility dynamic monitoring image information system can be established. Based on the theory of the measurement of the basic formula of visibility, the dynamic model of the optimization is established, set up 200 meters distance visual observation target system, research visual range detection algorithm process.
\end{abstract}

\section{Keywords}

The Highway, Visual Range, Dynamic Monitoring, Mathematical Model

\section{Introduction}

Fog is one of the most serious traffic accidents caused by highway disastrous weather. Over the past years, the highway traffic accidents caused by heavy fog occurred frequently. The visibility detection sample space is quite limited; it is difficult to truly reflect the visibility of the road section, which is not conducive to the analysis of fog distribution. Research on digital video camera provides distance measurement based on the new principle and method, and the way to obtain the target information is basically consistent with the observation of the human eye [1] [2]. The aim of this paper is to solve the problem of monitoring the visibility distance of 200 meters. According to the basic formula of photography measurement of visibility, to obtain the optimum value of the visibility calculation model, to make full use of the high density of highway traffic video camera device, a dynamic monitoring algorithm for visual safety distance of 
freeway is proposed.

\section{Dynamic Monitoring Image Information System}

Based on highway traffic video monitoring system of Expressway in Hebei province, Selected routes Zhangbei (plateau), Fengning (mountain), Xingtai (plain) and Laoting (coastal) (four counties in Hebei, China) four terrain environment, climate conditions of high-speed road section as a research test point, and the establishment of traffic video monitoring terminal system, set up regular image capture, image information of each traffic camera device.

\section{Visual Security Distance Database}

\subsection{Image Database}

In the process of visual safety distance monitoring, the system will continue to produce a large number of pictures, and therefore need to carry out scientific management of the picture [3]. The image data is the image pixel gray value of the record, to the rank data matrix representation. The image database is composed of two levels of image data and image data dictionary, and can be set according to the basic data of the image, retrieval and processing operation. Image database system can be used to deal with image data, graphics, graphics, data, general text, figures and other information [4].

\subsection{Observation Data Base}

Based on the SQL database technology, establish the visual range observation database. However, due to the complexity of the measurement between each other, it is often difficult to use accurate numerical methods and mathematical methods to determine [5]. According to the clarity of the image, the principle of fuzzy logic is applied, and observation data storage is analyzed, so as to obtain more accurate results for forecasting the visual safety distance on the highway.

\section{High Speed Road Distance Visual Safety Establishment}

Natural light is the more optional targets. Because the detection process of the human eye is a contrast detection process, the pattern of the target object should have the edge of high contrast [6]. The road visibility detection focuses within $200 \mathrm{~m}$ of the situation. Therefore, the distance information points are set at $20 \mathrm{~m}, 50 \mathrm{~m}, 100 \mathrm{~m}, 200 \mathrm{~m}$, as showed in Figure 1.

In the absence of natural light in the night, the luminous object light source is the ideal choice of visual distance. According to our provincial highway, according to the province of high-speed roads, traffic around the camera without self luminous body, the visible light from the reflected light traveling between the vehicle headlights and illuminated objects. The middle of the highway isolation fence as a target, on the one hand to ensure stability of the illuminated objects reflect light, to ensure the consistency of the target time on the other hand, avoid at different times due to atmospheric 


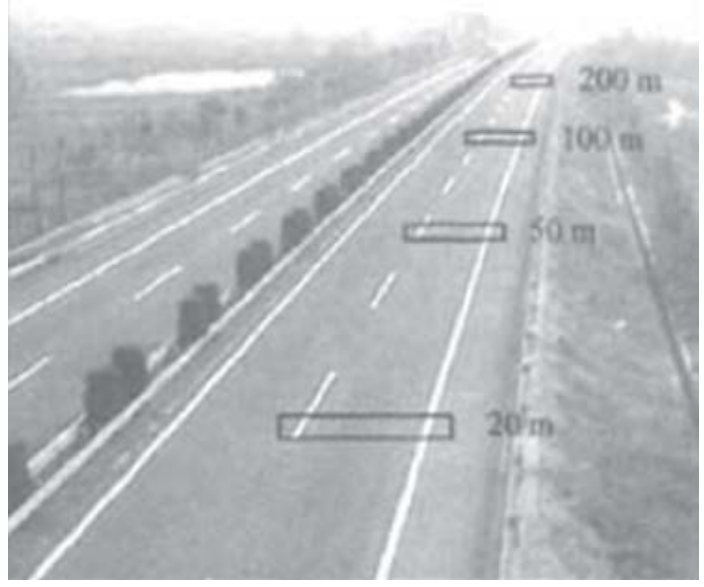

Figure 1. $200 \mathrm{~m}$ internal target selection.

changes caused by change of flux visibility misalignment [7]. Highway traffic monitoring cameras capture images as samples for target selection, shown in Figure 2.

For the daytime mist condition, the target object selection can be selected with the same night, that the middle of the highway guardrail. In the same section select the same night samples and found that the isolation barrier has become blurred, the difference of foreground and background luminance brightness is small, but the car lane on both sides but with a different background. In this case, select on both sides of road line as the target to determine the results as scheduled target quality control part in the calculation. The pictures are shown in Figure 3.

Quantitative measurement of visual distance by MOR, Incandescent lamp as the light source, the visible light spectrum natural, the characteristic is that it can cause the naked eye, and the normal human eyes can be different in the visible light at different wavelengths. The infrared and ultraviolet light has no visual response, according to the physical definition of meteorological optical range, establishment of test field visual distance target observation system, meet the visibility meter calibration and visual distance observation comparison.

\section{Calculation Model}

\subsection{Visibility Algorithm Flow (Figure 4)}

Visibility detection algorithm flow is as follows: establishment of observation target with distance information; eliminate the image pre-processing in image noise; target detection based on SAD algorithm; analog camera image edge feature extraction of human; nonlinear fitting of the relationship between the degree of contrast and distance, and the visibility value [8].

\subsection{Digital Photography Measurement Model of Visibility}

The camera and the pavement of highway traffic between the imaging geometry is the visibility value calculated on the basis of the model, as shown in Figure 5. 


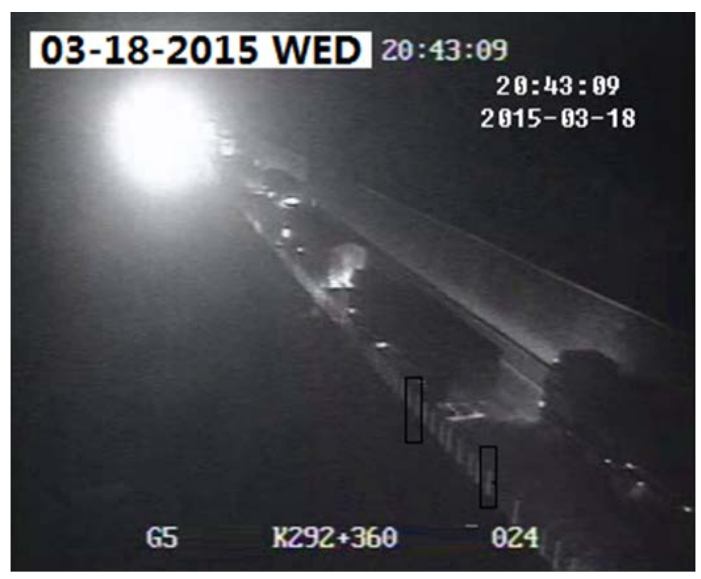

Figure 2. The target of the night fence.

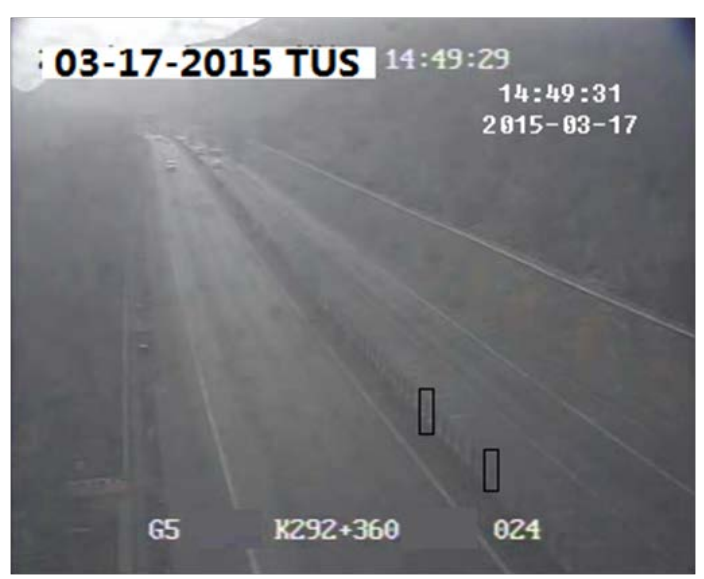

Figure 3. Lane targets under the mist.

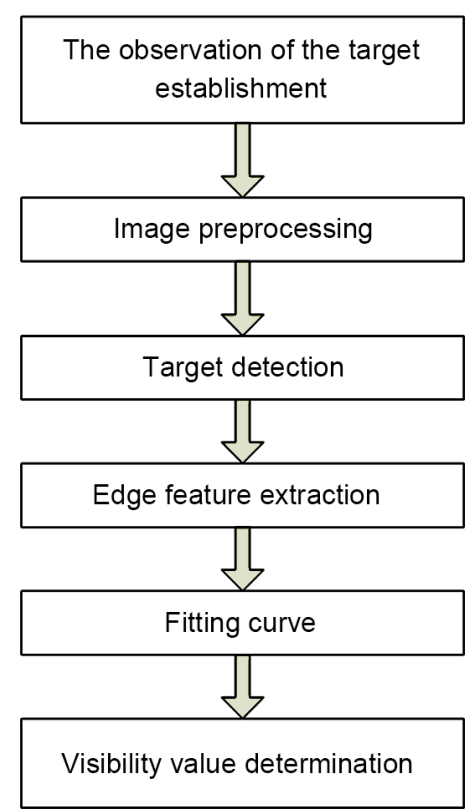

Figure 4. Visibility algorithm processes. 


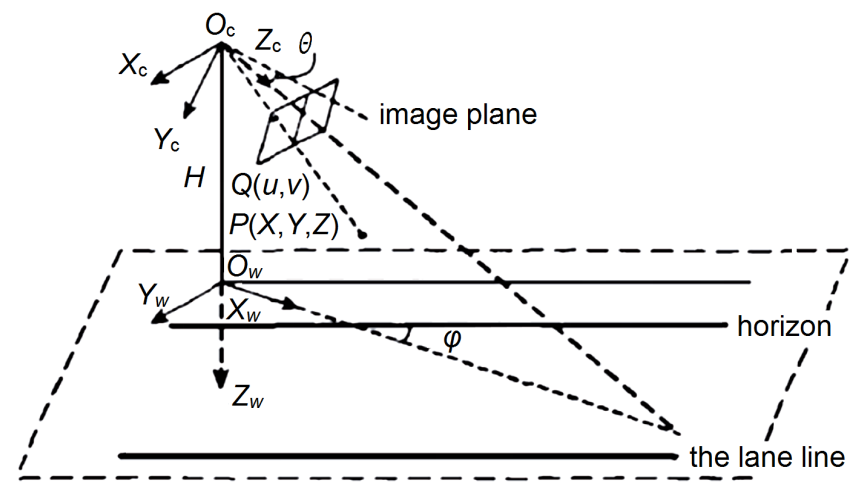

Figure 5. Visibility calculation models.

Based on the definition of camera parameters, the coordinate transformation between the ground coordinate system and the camera coordinate system is established, as follows:

$$
\left(\begin{array}{c}
X_{c} \\
Y_{c} \\
Z_{c} \\
1
\end{array}\right)=\left(\begin{array}{cccc}
0 & 1 & 0 & 0 \\
-\sin \theta & 0 & \cos \theta & H \cos \theta \\
-\cos \theta & 0 & \sin \theta & H \sin \theta \\
0 & 0 & 0 & 1
\end{array}\right)\left(\begin{array}{c}
X_{w} \\
Y_{w} \\
Z_{w} \\
1
\end{array}\right) .
$$

The distance from the camera on the actual road surface can be obtained by the above formula, as follows:

$$
D=\sqrt{X_{W}^{2}+Y_{W}^{2}} .
$$

Usually, a pixel normalized contrast $C<0.05$, that is, the pixel cannot be resolved by the human eye. Therefore, according to the results of the camera calibration, for all the pixels in the image contrast of $C \geq 0.05 i$, the distance between the camera and the camera can be calculated $d_{i}$. From the definition of visibility, the current value of the visibility $V=\max \left(d_{i}\right)$.

\subsection{Digital Photography Measurement of Visual Distance Model}

At night when there is no natural light of the light source, a luminous object is an ideal target visibility. The flow around the camera without self luminous body, the visible light source of the visible light source is from the reflected light between the moving vehicle lamp and the irradiated object. The middle of the highway guardrail as binocular object, on the one hand, the reflection coefficient and the scattering coefficient are in good agreement; on the other hand, it can eliminate the influence of the dark current and background stray light of the CCD digital camera system. The middle of the highway isolation fence as a target, establishes a numerical model of visual distance.

According to the law of Allard, the expression of visual distance at night is given:

$$
E_{t h}=\frac{I^{-\sigma \nu}}{V^{2}} .
$$


The luminance threshold value is when the visibility distance is $V$ and the intensity of the light source is $I$. Night visual distance affected by the flux density in the atmospheric attenuation. The above formula is being written as follows:

$$
V=\frac{1}{\sigma}\left(\ln \frac{1}{E_{t h}}-2 \ln V\right) .
$$

The visibility is calculated by the formula above.

It is obviously unrealistic to calculate the visibility of the above formula. It does not know about $\sigma$, and must be suitable for different lighting conditions. Therefore, the double object method is used to measure the visibility of the night [9]. This is no longer needed $E_{t h}$ and $\sigma$.

Select the middle of the highway as a binocular reference standard, visibility calculation model. The distance from the camera namely is $d_{1}$ and $d_{2}$. Its settings are shown in Figure 6.

Assuming monitoring of the barrier imaging brightness is $E_{1}$ and $E_{2}$. According to the above two type can have a visible distance of the night:

$$
V=\frac{\mathrm{d}_{1}-\mathrm{d}_{2}}{\ln \frac{E_{2} \mathrm{~d}_{2}^{2}}{E_{1} \mathrm{~d}_{1}^{2}}}(\varepsilon-2 \ln V) .
$$

$\varepsilon$ is determined by the field experiment. In order to eliminate the recurrence relation, it is further simplified:

$$
V \approx \frac{\left(\mathrm{d}_{1}-\mathrm{d}_{2}\right) \alpha}{\ln \frac{E_{2} \mathrm{~d}_{2}^{2}}{E_{1} \mathrm{~d}_{1}^{2}}} .
$$

$\alpha$ is a multiplicative factor, can be obtained through a large amount of data information, $d_{1}$ and $d_{2}$ are obtained by measuring, $E_{1}$ and $E_{2}$ are obtained by calculating [10]. The model can be established by referring to the model of the visual distance in the night.

\section{Conclusion}

The pavement and the two sides are directly observed from the view point of video observation, and the selection of the same kind of object as the object is selected. In this

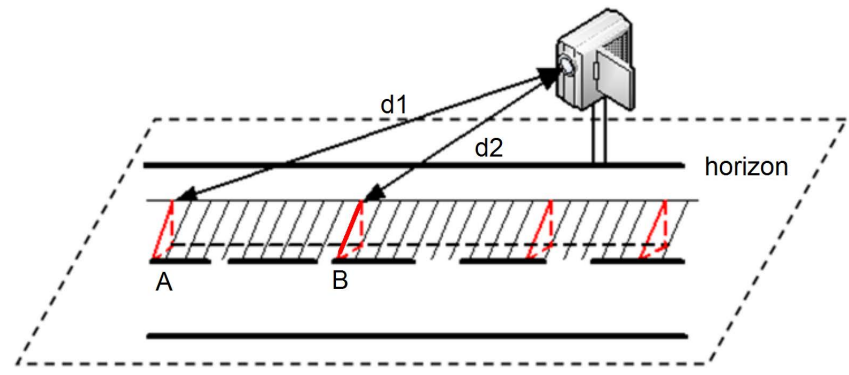

Figure 6. Double target selection schemes. The red triangle denotes the projection after irradiation. 
paper, the concept of binocular vision is introduced to minimize the influence of the atmospheric extinction coefficient and the brightness threshold, and dynamic monitoring of visual safe distance in the 200 meters of the highway is solved. The project can build the expressway visual distance extension prediction products based on $50 \mathrm{~m}, 100$ $\mathrm{m}$ and $200 \mathrm{~m}$ visual distance forecasting methods, and establish the short-term forecast service products within 3 hours of the expressway to improve the quality of forecasting. After the completion, the project can be extended to neighboring provinces and cities.

\section{References}

[1] Lin, M. and Zhang, W. (2008) Setting Methods of Traffic Signs on Expressway. Communications Standardization, 9, 31-33.

[2] Guazzi, A.R., Villarroel, M., Jorge, J., Daly, J., Frise, M.C., Robbins, P.A., et al. (2015) Non-Contact Measurement of Oxygen Saturation with an RGB Camera. Biomedical Optics Express, 6, 3320-3338. http://dx.doi.org/10.1364/BOE.6.003320

[3] Hyde, R.F. (2014) Safety Distance Monitoring of Adjacent Vehicles. US Patent No. 20140095061.

[4] Bian, M. and Li, K. (2005) Estimation of Longitudinal Road Friction Coefficient Based on a Dual-Exponential Model. Transactions of the Chinese Society of Agricultural Machinery, 36, 5-8.

[5] You, F., Zhang, R., Wang, H., et al. (2013) Warning Model for Safety Analysis of Overtaking Behavior Based on Longitudinal Safety Spacing. Journal of South China University of Technology (Natural Science Edition), 41, 87-92.

[6] Xu, L., Ni, Y., Luo, Q., et al. (2011) Lane-Changing Model Based on Minimum Safety Distance. Journal of Guangxi Normal University: Natural Science Edition, 29, 1-6.

[7] Xu, H., Pei, Y. and Cheng, G. (2010) Study on the Safety of Lane-Changing Based on Virtual Desire Trajectory. China Safety Science Journal, 20, 90-95.

[8] Xu, L., Luo, Q., Wu, J., et al. (2010) Study of Car-Following Model Based on Minimum Safety Distance. Journal of Highway and Transportation Research and Development, 27, 95-100.

[9] Li, W., Gao, D. and Duan, J. (2010) Research on Lane Change Model for Intelligent Vehicles. Journal of Highway and Transportation Research and Development, 27, 119-123.

[10] Hidas, P. (2005) Modelling Vehicle Interactions in Microscopic Simulation of Merging and Weaving. Transportation Research Part C: Emerging Technologies, 13, 37-62. http://dx.doi.org/10.1016/j.trc.2004.12.003 
Submit or recommend next manuscript to SCIRP and we will provide best service for you:

Accepting pre-submission inquiries through Email, Facebook, LinkedIn, Twitter, etc. A wide selection of journals (inclusive of 9 subjects, more than 200 journals)

Providing 24-hour high-quality service

User-friendly online submission system

Fair and swift peer-review system

Efficient typesetting and proofreading procedure

Display of the result of downloads and visits, as well as the number of cited articles

Maximum dissemination of your research work

Submit your manuscript at: http://papersubmission.scirp.org/

Or contact gep@scirp.org 